\title{
Scale breaking and fluid dynamics in a dilute two-dimensional Fermi gas
}

\author{
Clifford Chafin and Thomas Schäfer \\ Department of Physics, North Carolina State University, Raleigh, NC 27695
}

\begin{abstract}
We study two observables related to the anomalous breaking of scale invariance in a dilute two dimensional Fermi gas, the frequency shift and damping rate of the monopole mode in a harmonic confinement potential. For this purpose we compute the speed of sound and the bulk viscosity of the two dimensional gas in the high temperature limit. We show that the anomaly in the speed of sound scales as $\left(2 P-\rho c_{s}^{2}\right) / P \sim z /\left[\log \left(T / E_{B}\right)\right]^{2}$, and that the bulk viscosity $\zeta$ scales as $\zeta / \eta \sim z^{2} /\left[\log \left(T / E_{B}\right)\right]^{6}$. Here, $P$ is the pressure, $c_{s}^{2}$ is the speed of sound, $\eta$ is the shear viscosity, $z$ is the fugacity, and $E_{B}$ is the two-body binding energy. We show that our results are consistent with the experimental results of Vogt et al. [Phys. Rev. Lett. 108, 070404 (2012)]. Vogt et al. reported a frequency shift $\delta \omega / \omega$ of the order of a few percent, and a damping rate smaller than the background rate $\Gamma / \omega_{0} \sim 5 \%$.
\end{abstract}




\section{INTRODUCTION}

Scale invariant or nearly scale invariant fluids play a role in many areas of physics. Examples include the three dimensional Fermi gas at unitary, the quark gluon plasma at very high temperature, and a number of fluids that can be described in terms of holographic dualities [1, 2]. A very interesting example is provided by a two-dimensional gas of fermions interacting via a zero range interaction. This system is scale invariant at the classical level, but scale invariance is broken in the quantum theory. The quantum mechanical scattering amplitude depends logarithmically on a scale, which we can take to be the binding energy $E_{B}$ of the two-body bound state. This is analogous to what happens in QCD in three dimensions. QCD is classically scale invariant, but at the quantum level scale invariance is broken and the coupling depends logarithmically on the QCD scale parameter.

Two properties related to the breaking of scale invariance were recently studied by Vogt et al. [3]. The first is the frequency of the monopole mode in a harmonically trapped gas. One can show that in a scale invariant gas this mode has frequency $2 \omega_{0}$, where $\omega_{0}$ is the frequency of the harmonic confinement potential [4]. Deviations from this value provide a measure of scale breaking [5, 6]. Vogt et al. found that these deviations are small, on the order of a few percent, for the entire range of parameters studied in their experiment. The second observable is the damping of the monopole mode. In a scale invariant fluid the monopole mode is undamped [7]. The experiments find the that the damping is too small to be reliably measured, although one has to keep in mind that the background damping rate is sizeable, $\Gamma \simeq 0.05 \omega_{0}$.

In this work we present a rigorous calculation of the frequency shift and the damping rate of the monopole mode in the high temperature limit. Our calculation is based on the virial expansion for thermodynamic properties, and on kinetic theory for non-equilibrium effects. We will show that the results are in agreement with the measured frequency shift, and consistent with the failure of the experiment to observe a non-zero damping rate. We note that the experimental data were taken for $T / T_{F} \sim 0.4$ and a range of values of $\log \left(T_{F} / E_{B}\right)$. In the vicinity of the BCS/BEC crossover, corresponding to $\log \left(T_{F} / E_{B}\right) \sim 0$, it is not clear that a high temperature calculation is quantitatively reliable. Ultimately comparison between theory and experiment will determine in what range of $T / T_{F}$ the kinetic theory description is applicable. In the case of the three-dimensional Fermi gas at unitarity there 
is some evidence that kinetic theory is reliable for $T \gtrsim 0.4 T_{F}$, see for example [8].

Our study builds on earlier work that relates the frequency shift of the monopole mode to scale breaking in the speed of sound [5], and on our own work on bulk viscosity in the three dimensional Fermi gas [9]. In the latter work we showed that the bulk viscosity of the three dimensional Fermi gas near unitarity scales as $\zeta \sim[(\Delta P) / P]^{2} \eta$, where $\Delta P=P-\frac{2}{d} \mathcal{E}$ is the scale breaking part of the pressure and $\eta$ is the shear viscosity. Here, $d$ is the number

of spatial dimensions and $\mathcal{E}$ is the energy density. We will show that the bulk viscosity of the two dimensional gas in the limit $T \gg E_{B}$ is even smaller than this estimate suggests. We find that $\zeta$ is suppressed by two additional powers of $\log \left(T / E_{B}\right)$.

This paper is organized as follows. In Sect. II introduce a diagrammatic approach to the virial expansion. We also apply this method to the calculation of the quasi-particle energy. In Sect. III we compute the frequency of the monopole mode. In Sect. IV we describe a calculation of the bulk viscosity in kinetic theory. We use the result to compute the damping of the monopole mode. We present an outlook in Sect. V.

\section{EQUILIBRIUM AND QUASI-PARTICLE PROPERTIES}

\section{A. Two body interaction}

A dilute gas of non-relativistic spin $1 / 2$ fermions can be described by the effective lagrangian

$$
\mathcal{L}=\psi^{\dagger}\left(i \partial_{0}+\frac{\nabla^{2}}{2 m}\right) \psi-\frac{C_{0}}{2}\left(\psi^{\dagger} \psi\right)^{2}
$$

where $m$ is the mass of the fermion and $C_{0}$ is the coupling constant. The scattering amplitude in the spin singlet channel is

$$
\mathcal{A}(E)=\frac{1}{C_{0}^{-1}-\Pi(E)}
$$

where $\Pi(E)$ is given by

$$
\Pi(E)=\int \frac{d^{2} q}{(2 \pi)^{2}} \frac{1}{E-\frac{q^{2}}{m}+i \epsilon} .
$$

This can be compared to the general structure of the s-wave scattering amplitude in two dimensions [10]

$$
\mathcal{A}(E)=\frac{4 \pi}{m} \frac{1}{-\pi \cot \delta(E)+i \pi}
$$


where $\delta(E)$ is the s-wave scattering phase shift. We conclude that $\cot \delta(E)=\frac{1}{\pi} \log \left(\frac{E}{E_{B}}\right)$ where $E_{B}$ is the two body binding energy. We also define the scattering length $a$ by $E_{B}=$ $1 /\left(m a^{2}\right)$. The relation between $C_{0}$ and $E_{B}$ depends on the regularization scheme. In cutoff regularization we find

$$
\frac{1}{C_{0}(\Lambda)}=\frac{m}{4 \pi} \log \left(\frac{m E_{B}}{\Lambda^{2}}\right)
$$

\section{B. Thermodynamic potential}

In order to compute the thermodynamic potential it is useful to apply a HubbardStratonovich transformation to the effective lagrangian. Introducing a complex di-fermion field $\Phi$ we can write

$$
\mathcal{L}=\psi^{\dagger}\left(i \partial_{t}+\frac{\nabla^{2}}{2 m}\right) \psi+\left[\left(\psi \sigma_{+} \psi\right) \Phi+\text { h.c. }\right]+\frac{1}{C_{0}}|\Phi|^{2}
$$

where $\sigma_{+}$is the Pauli spin raising matrix. The integration over the fermion fields is Gaussian. We obtain an effective action for the bosonic field $\Phi$,

$$
S=-\operatorname{Tr}\left[\log \left(G^{-1}\left[\Phi, \Phi^{*}\right]\right)\right]+\int d^{4} x \frac{1}{C_{0}}|\Phi|^{2}
$$

where $G^{-1}$ is a $2 \times 2$ matrix

$$
G^{-1}\left[\Phi, \Phi^{*}\right]=\left(\begin{array}{cc}
i \partial_{t}+\frac{\nabla^{2}}{2 m} & \Phi^{*} \\
\Phi & i \partial_{t}-\frac{\nabla^{2}}{2 m}
\end{array}\right)
$$

The thermodynamic potential $\Omega$ is computed using the Matsubara formalism. We continue the fields to imaginary time $\tau$ and impose periodic/anti-periodic boundary conditions on the bosonic/fermionic fields. We also introduce a chemical potential for $\psi$. We evaluate the partition function by expanding the logarithm in powers of $\Phi$. The leading term is the free fermion loop,

$$
\Omega_{1}=\frac{\nu z T}{\lambda^{2}}\left(1-\frac{z}{2}+O\left(z^{2}\right)\right)
$$

where $\nu=2$ is the number of degrees of freedom, $z=\exp (\mu / T)$ is the fugacity, and $\lambda=[(2 \pi) /(m T)]^{1 / 2}$ is the thermal wave length. Terms of order $z^{2}$ and higher arise from

quantum statistics. The complete $O\left(z^{2}\right)$ result includes quadratic fluctuations in $\Phi$. We find

$$
\Omega_{2}=T \sum_{n} \int \frac{d^{2} q}{(2 \pi)^{2}} \log \left[\mathcal{D}^{-1}\left(i \omega_{n}, q\right)\right]
$$


where $\omega_{n}=2 \pi n T$ are bosonic Matsubara frequencies and $\mathcal{D}^{-1}\left(\omega_{n}, q\right)$ is the one loop difermion polarization function

$$
\mathcal{D}^{-1}\left(i \omega_{n}, q\right)=\int \frac{d^{2} k}{(2 \pi)^{2}}\left\{\frac{1-f_{k}-f_{k+q}}{i \omega_{n}-\xi_{k}-\xi_{k+q}}-\frac{1}{E_{B}-2 \epsilon_{k}}\right\} .
$$

Here, $f_{k}=\left[\exp \left(\beta \xi_{k}\right)+1\right]^{-1}$ is the Fermi-Dirac distribution, $\xi_{k}=\epsilon_{k}-\mu$ and $\epsilon_{k}=k^{2} /(2 m)$. In order to deriving equ. (11) we have used the relation between $C_{0}$ and $E_{B}$ given in equ. (5). The sum over Matsubara frequencies in equ. (10) can be performed using contour integration. The result can be expressed in terms of the discontinuity of $\mathcal{D}^{-1}(\omega, q)$ along the real axis in the complex frequency plane. We obtain

$$
\Omega_{2}=\frac{1}{2 \pi i} \int_{-\infty}^{\infty} d \omega \int \frac{d^{2} k}{(2 \pi)^{2}} d i s c\left[\log \mathcal{D}^{-1}(\omega+i \epsilon, k)\right] f_{B E}(\omega)
$$

where $f_{B E}(\omega)=[\exp (\beta \omega)-1]^{-1}$ is the Bose-Einstein distribution function. In order to compute $\Omega_{2}$ at second order in fugacity $z$ we need to evaluate $\mathcal{D}^{-1}(\omega, k)$ to zeroth order in $z$. We get

$$
\mathcal{D}^{-1}(\omega, k)=\frac{m}{4 \pi} \log \left(-\frac{\omega-\frac{\epsilon_{k}}{2}+2 \mu}{E_{B}}\right) .
$$

The simplest strategy to compute the integral over $\omega$ and $k$ is to compute $n=(\partial \Omega) /(\partial \mu)$, and then integrate over $\mu$. The result can be used to extract the interaction part of the second virial coefficient. We find

$$
\delta b_{2}=e^{\beta E_{B}}-2 \int \frac{d k}{k} \frac{e^{-2 \beta \epsilon_{k}}}{\left[\log \left(a^{2} k^{2}\right)\right]^{2}+\pi^{2}},
$$

with $\beta=1 / T$. The result agrees with the expectation from the standard Beth-Uhlenbeck expression for the second virial coefficient in terms of the phase shift,

$$
\delta b_{2}=e^{\beta E_{B}}+\frac{1}{\pi} \int d k\left(\frac{d \delta}{d k}\right) e^{-2 \beta \epsilon_{k}}
$$

The integral can be computed in terms of a function called $\nu(x)$ in [11]. We find

$$
\delta b_{2}(T)=\nu\left(\beta E_{B}\right), \quad \nu(x)=\int_{0}^{\infty} \frac{x^{t} d t}{\Gamma(t+1)} .
$$

For small $x$ the function $\nu(x)$ can be expanded in inverse powers of $\log (1 / x)$, see App. B and [12]. This expansion determines the virial coefficient in the limit $T \gg E_{B}$. We get

$$
\delta b_{2}(T)=\frac{1}{\log \left(T / E_{B}\right)}+\frac{\gamma_{E}}{\left[\log \left(T / E_{B}\right)\right]^{2}}+\ldots
$$

where $\gamma_{E}$ is Euler's constant. 


\section{Quasi-particle properties}

We can construct a systematic expansion for the two-dimensional gas in the dilute limit by writing the lagrangian in terms of fermion and boson degrees of freedom. For this purpose we write the lagrangian as the sum of free and interacting terms, $\mathcal{L}=\mathcal{L}_{0}+\mathcal{L}_{1}$, with

$$
\begin{aligned}
& \mathcal{L}_{0}=\psi^{\dagger} \mathcal{S}^{-1}(\omega, p) \psi+\Phi^{*} \mathcal{D}^{-1}(\omega, p) \Phi, \\
& \mathcal{L}_{1}=\left[\left(\psi \sigma_{+} \psi\right) \Phi+h . c .\right]+\Phi^{*}\left[C_{0}^{-1}-\mathcal{D}^{-1}(\omega, p)\right] \Phi .
\end{aligned}
$$

Here, $\mathcal{S}(\omega, p)=\left[\omega-\xi_{p}\right]^{-1}$ is the fermion propagator and $\mathcal{D}(\omega, p)$ is the boson propagator given in equ. (13). The bosonic term in the interaction serves as a counterterm that removes fermion loop insertions in the boson self energy order by order in the expansion. The leading order fermion self energy is given by

$$
\Sigma\left(i \omega_{m}, k\right)=T \sum_{n} \int \frac{d^{2} q}{(2 \pi)^{2}} \mathcal{D}\left(i \omega_{n}+i \omega_{m}, q+k\right) \mathcal{S}\left(i \omega_{n}, q\right) .
$$

The Matsubara sum can be performed as in the previous section. The resulting contour integral receives contributions from the cut in the boson propagator and the pole in the fermion propagator. At leading order in the fugacity expansion we can neglect the cut contribution. In order to compute the quasi-particle self energy we analytically continue the pole term to the on-shell point $i \omega_{m}=\xi_{k}$. The result can be determined analytically as an expansion in $\left[\log \left(T / E_{B}\right)\right]^{-1}$. We get

$$
\Sigma(k)=-\frac{2 z T}{\log \left(T / E_{B}\right)}\left\{1-\frac{1}{\log \left(T / E_{B}\right)}\left[-E i\left(-\frac{\epsilon_{k}}{T}\right)+\log \left(-\frac{\epsilon_{k}}{2 T}\right)-i \pi\right]+\ldots\right\},
$$

where $E i$ is the exponential integral. The quasi-particle energy is $E_{k}=\epsilon_{k}+\operatorname{Re} \Sigma(k)$ and the width is $\Gamma_{K}=-\operatorname{Im} \Sigma(k)$. We note that $\operatorname{Re} \Sigma$ is momentum independent at leading order in $\left[\log \left(T / E_{B}\right)\right]^{-1}$, but develops momentum dependence at next-to-leading order.

\section{FREQUENCY SHIFT OF THE MONOPOLE MODE}

We follow the work of [5, 13, 14] and compute the frequency of the monopole mode using a variational method. The use of variational methods in hydrodynamics was pioneered in $[15,16]$. The Euler equations follow from the variational principle $\delta S=0$ with $S=\int d t L$ and

$$
L=\int d^{2} r\left[\frac{1}{2} \rho \vec{u}^{2}-\mathcal{E}(\rho, \bar{s})-\frac{\rho}{m} V_{e x t}(r)-\phi\left(\frac{\partial \rho}{\partial t}+\vec{\nabla} \cdot(\vec{u} \rho)\right)-\xi\left(\frac{\partial \rho \bar{s}}{\partial t}+\vec{\nabla} \cdot(\vec{u} \rho \bar{s})\right)\right] .
$$


The hydrodynamic variables are the mass density $\rho$, the velocity $\vec{u}$, and the entropy per particle $\bar{s}=s / n$. Here, $s$ is the entropy density and $n$ is the particle density. The fields $\phi$ and $\xi$ are Lagrange multipliers that enforce the continuity and entropy conservation equations. $\mathcal{E}(\rho, \bar{s})$ is the energy density and $V_{e x t}$ is an external potential. We will consider a harmonic, rotationally invariant potential $V_{e x t}=\frac{1}{2} m \omega_{0} \vec{r}^{2}$. We also add Lagrange multipliers $\mu_{0}$ and $T_{0}$ to allow for solutions with finite particle number and entropy,

$$
L^{\prime}=L+\frac{1}{m} \int d^{2} r\left(\rho \mu_{0}+\rho \bar{s} T_{0}\right)
$$

Varying $S^{\prime}$ with respect to the hydrodynamic variables and setting $\vec{u}=0$ we get the hydrostatic equations

$$
\begin{aligned}
& \vec{\nabla} P=\left.\frac{\partial P}{\partial \rho}\right|_{\bar{s}} \vec{\nabla} \rho+\left.\frac{\partial P}{\partial \bar{s}}\right|_{\rho} \vec{\nabla} \bar{s}=-n \vec{\nabla} V_{e x t}, \\
& \vec{\nabla} T=\left.\frac{\partial T}{\partial \rho}\right|_{\bar{s}} \vec{\nabla} \rho+\left.\frac{\partial T}{\partial \bar{s}}\right|_{\rho} \vec{\nabla} \bar{s}=0 .
\end{aligned}
$$

The partial derivatives are related by the Maxwell relation

$$
\left.\rho \frac{\partial T}{\partial \rho}\right|_{\bar{s}}=\left.\frac{1}{\rho} \frac{\partial P}{\partial \bar{s}}\right|_{\rho}
$$

We will denote the solution to the hydrostatic equations by $\rho_{0}$ and $\bar{s}_{0}$. Small oscillations around this solution are governed by

$$
L_{2}=\frac{1}{2} \int d^{2} r\left[\rho_{0} \vec{u}^{2}-\frac{1}{\rho_{0}}\left(\frac{\partial P}{\partial \rho}\right)_{\bar{s}}(\delta \rho)^{2}-2 \rho_{0}\left(\frac{\partial T}{\partial \rho}\right)_{\bar{s}} \delta \rho \delta \bar{s}-\rho_{0}\left(\frac{\partial T}{\partial \bar{s}}\right)_{\rho}(\delta \bar{s})^{2}\right] .
$$

A variational ansatz for monopole vibrations is given by $\vec{u}=\vec{u}_{0} e^{-i \omega t}$ with $\vec{u}_{0}=\alpha \vec{r}$. This ansatz corresponds to an exact solution of the Euler equation in the scale invariant case. The continuity equation implies that

$$
\delta \rho=-\frac{i}{\omega} \vec{\nabla} \cdot\left(\rho_{0} \vec{u}_{0}\right), \quad \delta \bar{s}=-\frac{i}{\omega} \vec{u}_{0} \cdot \vec{\nabla}\left(\bar{s}_{0}\right) .
$$

The variational estimate for the mode frequency $\omega$ is obtained by setting $L_{2}$ to zero. Using the hydrostatic equations (23, 24) and the Maxwell relation (25) we find [5]

$$
\frac{\omega^{2}}{4 \omega_{0}^{2}}=1-\frac{1}{2}\left[\int d^{2} r \gamma_{2}(r)\right] /\left[\int d^{2} r n_{0}(r) V_{e x t}(r)\right]
$$

with $\gamma_{2}=2 P-\rho c_{s}^{2}$, where $c_{s}^{2}$ is the speed of sound. This result has a simple physical interpretation: The deviation of the monopole frequency from the value $\omega=2 \omega_{0}$ in a scale 


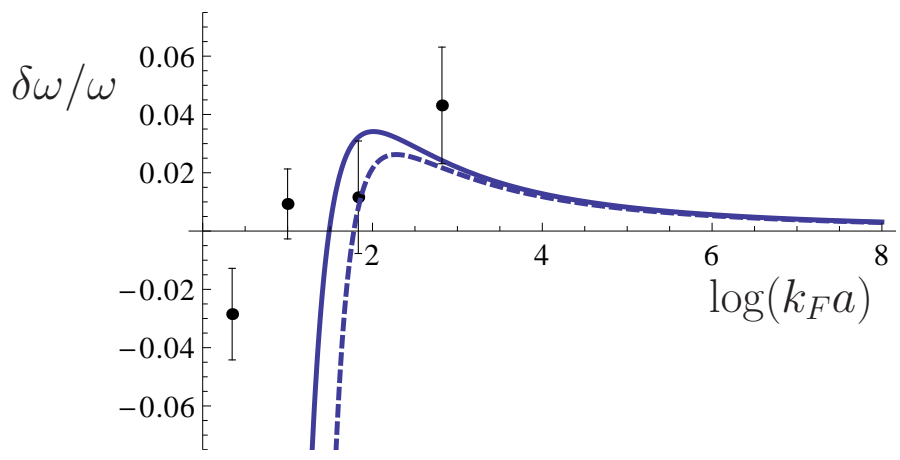

FIG. 1: Frequency shift relative to the prediction $\omega=2 \omega_{0}$ for a scale invariant fluid of the collective monopole mode in a two dimensional Fermi gas. The frequency shift is shown as a function of $\log \left(k_{F} a\right)$ for $T / T_{F}=0.42$. The solid line is based on the virial expansion, the dashed line shows the result at leading order in $1 /\left[\log \left(T / E_{B}\right)\right]$, and the data are taken from Fig. 1c in [3]

invariant theory [4] is governed by the trap average of the difference between the actual speed of sound and the sound speed $c_{s, 0}^{2}=2 P / \rho$ in a scale invariant gas.

In the dilute limit we can compute $\gamma_{2}$ using the virial equation of state. We find

$$
\gamma_{2}=\frac{m z^{2} E_{B}^{2}}{\pi} \nu^{\prime \prime}\left(\frac{E_{B}}{T}\right) .
$$

We perform the trap average using the density profile in the high temperature limit,

$$
n_{0}(x)=\frac{m T}{2 \pi}\left(\frac{T_{F}}{T}\right)^{2} \exp \left(-\frac{m \omega_{0}^{2} r^{2}}{2 T}\right),
$$

where $T_{F}=\sqrt{N} \omega_{0}$ is the Fermi temperature of the trap. We can now compute the frequency shift in the dilute limit

$$
\frac{\omega^{2}}{4 \omega_{0}^{2}}=1-\frac{1}{8} \frac{T_{F}^{2} E_{B}^{2}}{T^{4}} \nu^{\prime \prime}\left(\frac{E_{B}}{T}\right) .
$$

The result is shown in Fig. 1, We observe that the frequency shift is in agreement with the data for $\log \left(k_{F} a\right) \gtrsim 1.75$. For smaller values of $\log \left(k_{F} a\right)$ the contribution of the bound state is large and the virial expansion breaks down. In Fig. 1 we also show the contribution of the leading $\left[\log \left(T / E_{B}\right)\right]^{-1}$ term in the second virial coefficient. We observe that the leading term dominates for $\log \left(k_{F} a\right) \gtrsim 2$. 


\section{BULK VISCOSITY AND THE DAMPING OF THE MONOPOLE MODE}

\section{A. Chapman-Enskog expansion}

In the context of hydrodynamics the damping of the monopole mode is determined by the bulk viscosity of the two dimensional Fermi gas. In this section we will use kinetic theory and the Chapman-Enskog expansion to compute the bulk viscosity. An analogous calculation of the shear viscosity and the damping of the quadrupole mode can be found in [17-19].

In kinetic theory we write the stress tensor of the gas in terms of the quasi-particle distribution function $f_{p}(\vec{x}, t)$. We will assume that the gas is spin-symmetric so that $f_{p}^{\uparrow}=$ $f_{p}^{\downarrow} \equiv f_{p}$. Close to equilibrium we can write

$$
f_{p}(\vec{x}, t)=f_{p}^{0}(\vec{x}, t)+\delta f_{p}(\vec{x}, t)=f_{p}^{0}(\vec{x}, t)\left(1-\frac{\psi_{p}}{T}\right),
$$

where $f_{p}^{0}(\vec{x}, t)$ is the local equilibrium distribution in a fluid with local velocity $\vec{u}(\vec{x}, t)$, temperature $T(\vec{x}, t)$ and chemical potential $\mu(\vec{x}, t)$. In the case of a fluid undergoing a scaling expansion the off-equilibrium factor has the form $\psi_{p}=\chi^{B}(\vec{p}) \vec{\nabla} \cdot \vec{u}$. The off-equilibrium distribution $\delta f_{p}$ induced by the bulk stress $\vec{\nabla} \cdot \vec{u}$ is determined by the Boltzmann equation

$$
D f_{p} \equiv\left(\frac{\partial}{\partial t}+\vec{v}_{p} \cdot \vec{\nabla}_{x}+\vec{F} \cdot \vec{\nabla}_{p}\right) f_{p}(\vec{x}, t)=C\left[f_{p}\right]
$$

Here, $\vec{v}_{p}=\vec{\nabla}_{p} E_{p}$ is the quasi-particle velocity, $\vec{F}=-\vec{\nabla}_{x} E_{p}$ is the force term, and $C\left[f_{p}\right]$ is the collision term. Using the methods described in [9] the left hand side of the Boltzmann equation can be written as

$$
\frac{T}{f_{0}} D f_{0}=\left\{\frac{\alpha \rho c_{T}^{2}}{c_{V}} h-m c_{s}^{2}+\left[\frac{1}{2} \vec{p} \cdot \vec{\nabla}_{p}-\frac{\alpha \rho c_{T}^{2}}{c_{V}}+\left.\rho c_{s}^{2} \frac{\partial}{\partial P}\right|_{T}+\left.\frac{\alpha \rho c_{T}^{2}}{c_{V}} T \frac{\partial}{\partial T}\right|_{P}\right] E_{p}\right\} \vec{\nabla} \cdot \vec{u}
$$

where $h$ is the enthalpy per particle, $c_{s} / c_{T}$ are the speed of sound at constant entropy per particle/temperature, $c_{V}$ is the specific heat at constant volume, and $\alpha$ is the thermal expansion coefficient. This result can be simplified by writing $E_{p}=\epsilon_{p}+\Delta E_{P}$ and dropping terms of order $z^{2}$. We get

$$
\begin{aligned}
\frac{T}{f_{0}} D f_{0}= & \left\{\frac{\alpha \rho c_{T}^{2}}{c_{V}} h-m c_{s}^{2}+\left[1-\frac{\alpha \rho c_{T}^{2}}{c_{V}}\right] \epsilon_{p}\right. \\
& \left.+\left[\frac{1}{2} \vec{p} \cdot \vec{\nabla}_{p}+\mu \frac{\partial}{\partial \mu}+T \frac{\partial}{\partial T}-1\right] \Delta E_{p}\right\} \vec{\nabla} \cdot \vec{u}
\end{aligned}
$$


This result satisfies a number of consistency checks. Equ. (35) vanishes for a free gas, and for a general scale invariant gas characterized by a temperature independent second virial coefficient and a scale invariant dispersion law of the form $\Delta E_{p} \sim z T g\left(\epsilon_{p} / T\right)$ where $g(x)$ is an arbitrary function.

In order to solve the Boltzmann equation in the limit $T \gg E_{B}$ we use the second virial coefficient given in equ. (16) and the quasi-particle energy using equ. (20). We expand all quantities to leading non-trivial order in $\left[\log \left(T / E_{B}\right)\right]^{-1}$. We get $\frac{T}{f_{0}} D f_{0} \equiv X_{p}(\vec{\nabla} \cdot \vec{u})$ with

$$
X_{p}=\frac{2 z T}{\left[\log \left(T / E_{B}\right)\right]^{3}}\left\{\frac{\epsilon_{p}}{T}-\left(1+2 \gamma_{E}\right)+2\left(E i\left(-\frac{\epsilon_{p}}{T}\right)-\log \left(\frac{\epsilon_{p}}{2 T}\right)\right)\right\},
$$

where $E i(x)$ is the exponential Integral. We note that both the thermodynamic terms as well as the self energy contain contributions of order $\left[\log \left(T / E_{B}\right)\right]^{-2}$, but these terms cancel and $X_{p}$ scales as $\left[\log \left(T / E_{B}\right)\right]^{-3}$. Equ. (36) satisfies two sum rules

$$
\int d \Gamma_{p} f_{p}^{0} X_{p}=0, \quad \int d \Gamma_{p} f_{p}^{0} \epsilon_{p} X_{p}=0
$$

with $d \Gamma_{p}=d^{2} p /(2 \pi)^{2}$. The sum rules follow from conservation of particle number and energy.

\section{B. Collision term}

The linearized collision operator can be written as

$$
C\left[f_{p}^{0}+\delta f_{p}\right] \equiv \frac{f_{p}^{0}}{T} C_{L}\left[\chi_{B}(p)\right](\vec{\nabla} \cdot \vec{u})
$$

where, at leading order in the fugacity, the collision term is dominated by two-body collisions. We have

$$
C_{L}\left[\chi_{B}\left(p_{1}\right)\right]=\int\left(\prod_{i=2}^{4} d \Gamma_{i}\right) w(1,2 ; 3,4) f_{p_{2}}^{0}\left[\chi_{B}\left(p_{1}\right)+\chi_{B}\left(p_{2}\right)-\chi_{B}\left(p_{3}\right)+\chi_{B}\left(p_{4}\right)\right]
$$

where $w(1,2 ; 3,4)$ is the transition rate

$$
w(1,2 ; 3,4)=(2 \pi)^{3} \delta^{2}\left(\sum_{i} \vec{p}_{i}\right) \delta\left(\sum_{i} E_{i}\right)|\mathcal{A}|^{2}
$$

and $\mathcal{A}$ is the scattering amplitude given in equ. (2). In order to be consistent with the

calculation of the streaming term we expand the scattering amplitude to leading order in $\left[\log \left(T / E_{B}\right)\right]^{-1}$. We get

$$
\mathcal{A}=\frac{4 \pi}{m \log \left(T / E_{B}\right)} .
$$


To leading order in $z$ we can approximate the quasi-particle energy by the non-interacting result $E_{p} \simeq \epsilon_{p}$. The linearized Boltzmann equation

$$
X_{p}=C_{L}\left[\chi_{B}(p)\right]
$$

can be solved by expanding the off-equilibrium factor $\chi_{B}(p)$ in Laguerre polynomials

$$
\chi_{B}(p)=\sum_{i=2}^{N} c_{i} L_{i}^{0}\left(\frac{\epsilon_{p}}{T}\right) .
$$

Restricting the sum to terms of order $i \geq 2$ guarantees that the orthogonality constraints (37) are satisfied. The coefficients $c_{i}$ can be determined by taking moments of the Boltzmann equation (42) with $L_{i}^{0}\left(\epsilon_{p} / T\right)$ for $i=2, \ldots, N$. As a first approximation we can take $N=2$. We find

$$
\chi_{B}(p)=\frac{1}{4 \pi \log \left(T / E_{B}\right)}\left[2-4\left(\frac{\epsilon_{P}}{T}\right)+\left(\frac{\epsilon_{P}}{T}\right)^{2}\right] .
$$

The convergence of the expansion in Laguerre polynomials was studied in the case of three dimensions in [9]. We found that corrections to the leading term contribute to the bulk viscosity at a level of less than $5 \%$.

\section{Off-equilibrium stress and bulk viscosity}

In fluid dynamics the trace of the stress tensor in the fluid rest frame is given by $\Pi \equiv$ $\frac{1}{2} \Pi_{i i}=P-\zeta(\vec{\nabla} \cdot \vec{u})$, where $\zeta$ is the bulk viscosity. In kinetic theory this expression has to be matched against

$$
\Pi\left[f_{p}\right]=\frac{\nu}{2} \int d \Gamma_{p}\left(\vec{v}_{p} \cdot \vec{p} \cdot\right) f_{p}+\nu \int d \Gamma_{p} E_{p} f_{p}-\mathcal{E}\left[f_{p}\right],
$$

where $\nu=2$ is the number of spin degrees of freedom, $d \Gamma_{p}=d^{2} p /(2 \pi)^{2}$ is the phase space measure, $f_{p}=f(\vec{p}, \vec{x}, t)$ is the quasi-particle distribution function, $E_{p}$ is the quasiparticle energy and $\vec{v}_{p}=\vec{\nabla} E_{p}$ is the quasi-particle velocity. We split the distribution in an equilibrium and a non-equilibrium piece, $f_{p}=f_{p}^{0}+\delta f_{p}$, and write the bulk stress as

$$
\Pi\left[f_{p}^{0}+\delta f_{p}\right] \equiv \Pi\left[f_{p}^{0}\right]+\delta \Pi \equiv \Pi^{0}+\delta \Pi .
$$

The term $\delta \Pi$ is then identified with viscous correction $-\zeta(\vec{\nabla} \cdot \vec{u})$ in fluid dynamics. We compute $\Pi\left[f_{p}^{0}+\delta f_{p}\right]$ by functionally expanding equ. (45) in powers of $\delta f_{p}$. We find [9]

$$
\delta \Pi=\nu \int d \Gamma_{p} \delta f_{p}\left(\frac{1}{2} \vec{p} \cdot \vec{\nabla}_{p}+\mu \frac{\partial}{\partial \mu}+T \frac{\partial}{\partial T}-1\right) \Delta E_{p} .
$$


It is interesting to note that the bulk stress is determined by the same scale violating part of the self energy that appears in the streaming term (35). This ensures that in a scale invariant gas there is no bulk viscosity irrespective of the structure of the off-equilibrium distribution function.

We can now compute the bulk viscosity by inserting the solution of the linearized Boltzmann equation given in equ. (44) into the expression for the bulk stress. Comparing the result to $\delta \Pi=-\zeta(\vec{\nabla} \cdot \vec{u})$ determines the bulk viscosity. We find

$$
\zeta=\frac{1}{2 \pi} \frac{z^{2} \lambda^{-2}}{\left[\log \left(T / E_{B}\right)\right]^{4}} .
$$

This result is valid in the limit $z \ll 1$ and $\log \left(T / E_{B}\right) \gg 1$. Higher order corrections in $z$ require a calculation of the pressure at the level of the third virial coefficient, and the inclusion of three-body scattering in the collision term. Higher order terms in $\left[\log \left(T / E_{B}\right)\right]^{-1}$ can be determined by computing the self energy to all orders in in the logarithm of $T / E_{B}$, as we have done for the second virial coefficient. However, unless we include bosonic quasiparticles in the kinetic theory, the result for the bulk viscosity will still break down for $T \sim E_{B}$. We can compare equ. (48) to the result for the shear viscosity obtained in [17, 18]

$$
\eta=\frac{\lambda^{-2}}{\pi}\left[\log \left(T / E_{B}\right)\right]^{2},
$$

where we have taken the limit $\log \left(T / E_{B}\right) \gg 1$ used in the calculation of the bulk viscosity. We observe that $\zeta$ is suppressed by two additional powers of $\left[\log \left(T / E_{B}\right)\right]^{-1}$ compared to the expectation [9] $\zeta \sim[(\Delta P) / P]^{2} \eta$, where $\Delta P=P-\mathcal{E}$ is the scale breaking part of the equilibrium pressure. The reason for this extra suppression is related to the fact that the leading scale violating term in the quasi-particle energy is just a shift in the chemical potential, which does not contribute to the bulk pressure. Finally, we can write equ. (48) in terms of dimensionless ratios. We find

$$
\frac{\zeta}{n}=\frac{1}{4 \pi} \frac{1}{\left[\log \left(T / E_{B}\right)\right]^{4}}\left(\frac{T_{F}^{l o c}}{T}\right),
$$

where $T_{F}^{l o c}$ is the Fermi temperature of the homogeneous gas.

\section{Damping of monopole oscillations}

The damping of the monopole mode is determined by the amount of energy dissipated by viscous effects. Note that the cloud remains approximately isothermal so that thermal 


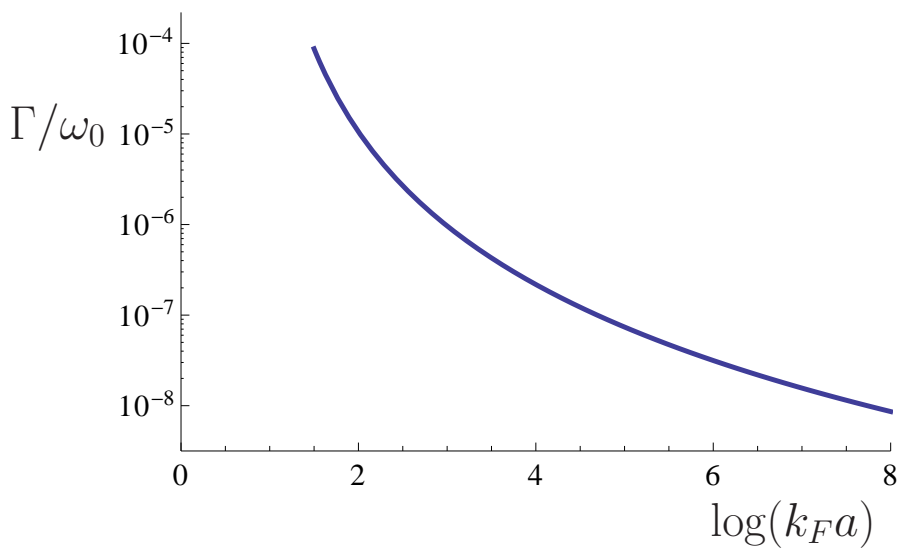

FIG. 2: Damping of the collective monopole mode in a $2 \mathrm{~d}$ Fermi gas. The figure shows $\Gamma / \omega_{0}$, the damping rate in units of the trap frequency, as a function of $\log \left(k_{F} a\right)$ for $T / T_{F}=0.42$ and $N=4 \cdot 10^{3}$.

conductivity does not contribute to dissipation. The rate of energy dissipation is

$$
\dot{E}=-\frac{1}{2} \int d^{2} r \zeta(r)(\vec{\nabla} \cdot \vec{u})^{2}
$$

and the damping constant is $\Gamma=-\dot{E} /(2 E)$, where $E$ is energy of the collective mode. Using the velocity profile $\vec{u} \sim \vec{r}$ of the monopole mode we find

$$
\Gamma=\left[\int d^{2} r \zeta(r)\right] /\left[m \int d^{2} x n_{0}(r) \vec{r}^{2}\right] \text {. }
$$

This result can be evaluated in the same way as the frequency shift considered in Sect. III, We get

$$
\frac{\Gamma}{\omega_{0}}=\frac{1}{32 \pi N^{1 / 2}} \frac{1}{\left[\log \left(T / E_{B}\right)\right]^{4}}\left(\frac{T_{F}}{T}\right)
$$

where $N$ is the number of particles and $T_{F}=\sqrt{N} \omega_{0}$ is the Fermi temperature of the trap. The result is plotted in Fig. 2. We consider the conditions explored in the experiment of Vogt et al., $N=4 \cdot 10^{3}$ and $T / T_{F}=0.42$. Based on our results for the frequency shift we assume that equ. (53) is reliable for $\log \left(k_{F} a\right) \gtrsim 2$. We observe that the damping constant in this regime is extremely small, $\Gamma / \omega_{0}<10^{-4}$. This is consistent with the measurements of Vogt et al., who find $\Gamma / \omega_{0}<5 \cdot 10^{-2}$, but our result implies that it will be very difficult to measure the bulk viscosity of the two dimensional gas in the BCS limit. 


\section{OUTLOOK}

The main results obtained in this work are the scaling of the pressure anomaly $(\Delta P) / P \sim$ $z /\left[\log \left(T / E_{B}\right)\right]^{2}$ and the bulk viscosity $\zeta / \eta \sim z^{2} /\left[\log \left(T / E_{B}\right)\right]^{6}$ in the high temperature limit $z \ll 1$ and $\log \left(T / E_{B}\right) \gg 1$. These results quantitatively explain the observed frequency shift of the monopole mode, and the failure of the experiment to detect a non-zero damping rate.

It would be interesting to extend the calculation to the regime $T \sim E_{B}$. In the context of kinetic theory this would presumably require the inclusion of explicit bosonic degrees of freedom. Alternatively, one might try to compute the pressure and the bulk viscosity using purely diagrammatic methods. At weak coupling the mechanism for generating bulk viscosity is related to a finite relaxation time for rearranging the internal energy of the gas among non-interacting and interacting terms in the single particle energy. For $T \sim E_{B}$ the physical mechanism is likely to be related to the formation of molecules. This involves three-body collisions, and is therefore suppressed in the low density limit $z \ll 1$, but the process may be enhanced by powers of $\log \left(T / E_{B}\right)$.

It is also important to consider the frequency dependence of the bulk viscosity. Taylor and Randeria proved the sum rule [5]

$$
\frac{2}{\pi} \int_{0}^{\infty} d \omega \zeta(\omega)=3 P-\mathcal{E}-\rho c_{s}^{2} .
$$

At high temperature the right hand side scales as $z^{2} m T^{2} /\left[\log \left(T / E_{B}\right)\right]^{3}$. On the left hand side

the contribution of the transport peak is $(\Delta \omega) z^{2} m T /\left[\log \left(T / E_{B}\right)\right]^{4}$, where $\Delta \omega$ is the width of the transport peak. Consistency with the sum rule then requires that $\Delta \omega \leq T \log \left(T / E_{B}\right)$. This bound can be compared to the width of the transport peak in the shear channel, which is $\Delta \omega \sim z T /\left[\log \left(T / E_{B}\right)\right]^{2} \ll T$. Taylor and Randeria also studied the tail of the spectral function (see also [20]). They find $\zeta(\omega) \sim z^{2} \lambda^{-2} T /\left(\omega\left[\log \left(\omega / E_{B}\right)\right]^{2}\left[\log \left(T / E_{B}\right)\right]^{2}\right.$ ), where we have used the high temperature limit of the contact. This result matches the kinetic theory prediction for $\omega \sim T$.

Acknowledgments: We thank John Thomas for useful discussions, and E. Taylor and M. Randeria for communications regarding their work. This work was supported in parts by the US Department of Energy grant DE-FG02-03ER41260. 


\section{Appendix A: Thermodynamics}

In this appendix we compute thermodynamic properties like the scale breaking contributions to the equation of state and the speed of sound using the virial equation of state. We follow the methods used in [9]. At second order in the virial expansion we have $P=\frac{\nu T}{\lambda^{2}}\left(z+b_{2}(T) z^{2}\right)$. At this order the scale breaking contribution to the equation of state is

$$
\frac{P-\mathcal{E}}{P}=-z T b_{2}^{\prime}(T)
$$

The enthalpy per particle is

$$
h=2 T\left[1-z\left(b_{2}(T)-\frac{1}{2} T b_{2}^{\prime}(T)\right)\right]
$$

and the specific heats are given by

$$
\begin{aligned}
& c_{V}=\frac{\nu z}{\lambda^{2}}\left[1+z\left(2 b_{2}(T)+T^{2} b_{2}^{\prime \prime}(T)\right)\right], \\
& c_{P}=c_{V}+\frac{\nu z}{\lambda^{2}}\left[1+z\left(4 b_{2}(T)-2 T b_{2}^{\prime}(T)\right)\right] .
\end{aligned}
$$

The speed of sound at constant $T$ and $s / n$ as well as the thermal expansion coefficient are

$$
\begin{aligned}
c_{T}^{2} & =\frac{T}{m}\left[1-2 z b_{2}(T)\right], \\
c_{s}^{2} & =\frac{2 T}{m}\left[1-z\left(b_{2}(T)+T b_{2}^{\prime}(T)+\frac{1}{2} T^{2} b_{2}^{\prime \prime}(T)\right)\right], \\
\alpha & =\frac{1}{T}\left[1+z\left(2 b_{2}(T)-T b_{2}^{\prime}(T)\right)\right] .
\end{aligned}
$$

Using these result we can compute the scale breaking parameter $\gamma_{2}$

$$
\gamma_{2}=2 P-\rho c_{s}^{2}=\frac{m T^{2} z^{2}}{\pi}\left(2 T b_{2}^{\prime}(T)+T^{2} b_{2}^{\prime \prime}(T)\right) .
$$

\section{Appendix B: Asymptotic expansion of $\nu(x)$}

The asymptotic expansion of $\nu(x)$ can be found by writing

$$
\nu(x)=\int_{0}^{\infty} d t \frac{\exp (-t \log (1 / x))}{\Gamma(t+1)},
$$

and Taylor expanding the gamma function, $\Gamma(t+1)^{-1}=1+\gamma_{E} t+\left(\frac{\gamma_{E}^{2}}{2}-\frac{\pi^{2}}{12}\right) t^{2}+O\left(t^{3}\right)$. We find

$$
\nu(x)=\frac{1}{\log (1 / x)}+\frac{\gamma_{E}}{[\log (1 / x)]^{2}}+\frac{\gamma_{E}^{2}-\frac{\pi^{2}}{6}}{[\log (1 / x)]^{3}}+\ldots
$$


We can now apply this results to the thermodynamic quantities studied in the first appendix. We find, in particular,

$$
\begin{aligned}
P-\mathcal{E} & =\frac{m T^{2} z^{2}}{\pi\left[\log \left(T / E_{B}\right)\right]^{2}}\left\{1+\frac{2 \gamma_{E}}{\log \left(T / E_{B}\right)}+\ldots\right\}, \\
\gamma_{2} & =-\frac{m T^{2} z^{2}}{\pi\left[\log \left(T / E_{B}\right)\right]^{2}}\left\{1+\frac{2 \gamma_{E}-2}{\log \left(T / E_{B}\right)}+\ldots\right\} .
\end{aligned}
$$

[1] T. Schäfer and D. Teaney, "Nearly Perfect Fluidity: From Cold Atomic Gases to Hot Quark Gluon Plasmas," Rept. Prog. Phys. 72, 126001 (2009) arXiv:0904.3107 [hep-ph]].

[2] A. Adams, L. D. Carr, T. Schäfer, P. Steinberg and J. E. Thomas, "Strongly Correlated Quantum Fluids: Ultracold Quantum Gases, Quantum Chromodynamic Plasmas, and Holographic Duality," New J. Phys. 14, 115009 (2012) arXiv:1205.5180 [hep-th]].

[3] E. Vogt, M. Feld, B. Fröhlich, D. Pertot, M. Koschorreck, M. Köhl, "Scale invariance and viscosity of a two-dimensional Fermi gas," Phys. Rev. Lett. 108, 070404 (2012) arXiv:1111.1173 [cond-mat.quant-gas].

[4] L. P. Pitaevskii, A. Rosch, "Breathing modes and hidden symmetry of trapped atoms in two dimensions," Phys. Rev. A 55, R853 (1997).

[5] E. Taylor, M. Randeria, "Apparent Low-Energy Scale Invariance in Two-Dimensional Fermi Gases," Phys. Rev. Lett. 109, 135301 (2012) [arXiv:1205.1525 [cond-mat.quant-gas]].

[6] J. Hofmann, "Quantum anomaly, universal relations and breathing mode of a two-dimensional Fermi gas," Phys. Rev. Lett. 108, 185303 (2012) arXiv:1112.1384 [cond-mat.quant-gas]].

[7] Y. Castin and F. Werner, "The Unitary Gas and its Symmetry Properties" in: Springer Lecture Notes in Physics "BEC-BCS Crossover and the Unitary FermHofmann:2011qsi gas," Wilhelm Zwerger (editor) [arXiv:1103.2851v2 [cond-mat.quant-gas]].

[8] T. Enss, R. Haussmann and W. Zwerger, "Viscosity and scale invariance in the unitary Fermi gas," Annals Phys. 326, 770 (2011) [arXiv:1008.0007 [cond-mat.quant-gas]].

[9] T. Schäfer and K. Dusling, "Bulk viscosity and conformal symmetry breaking in the dilute Fermi gas near unitarity," arXiv:1305.4688 [cond-mat.quant-gas].

[10] M. Randeria, J.-M. Duan, and L.-Y. Shieh, "Superconductivity in a two-dimensional Fermi gas: Evolution from Cooper pairing to Bose condensation," Phys. Rev. B 41, 327 (1990). 
[11] I. S. Gradshteyn, I. M.Ryzhik, "Table of Integrals, Series, and Products," Fifth Edition, Academic Press (1994).

[12] A. Erdelyi, "Higher Transcendetal Functions," Volume 3, McGraw Hill, New York (1955).

[13] E. Taylor, H. Hu, X.-J. Liu, and A. Griffin, "Variational theory of two-fluid hydrodynamic modes at unitarity," Phys. Rev. A 77, 033608 (2008) [arXiv:0711.0561 [cond-mat.other]].

[14] E. Taylor, H. Hu, X.-J. Liu, L. P. Pitaevskii, A. Griffin, S. Stringari, "First and second sound in a strongly interacting Fermi gas," Phys. Rev. A 80, 053601 (2009) arXiv:0905.0257 [condmat.quant-gas]].

[15] P. R. Zilsel, "Liquid Helium II: The Hydrodynamics of the Two-Fluid Model," Phys. Rev. 79, 309 (1950).

[16] H. Ito, "Variational Principle in Hydrodynamics," Prog. of Theor. Phys. 9, 117 (1953).

[17] T. Schäfer, "Shear viscosity and damping of collective modes in a two-dimensional Fermi gas," Phys. Rev. A 85, 033623 (2012) [arXiv:1111.7242 [cond-mat.quant-gas]].

[18] G. M. Bruun, "Shear viscosity and spin diffusion coefficient of a two-dimensional Fermi gas," Phys. Rev. A. 85, 013636 (2012) arXiv:1112.2395 [cond-mat.quant-gas]].

[19] S. K. Baur, E. Vogt, M. Köhl, G. M. Bruun, "Collective modes of a two-dimensional spin-1/2 Fermi gas in a harmonic trap," Phys. Rev. A. 87, 043612 (2013) arXiv:1301.0358 [condmat.quant-gas]].

[20] J. Hofmann, "Current response, structure factor and hydrodynamic quantities of a two- and three-dimensional Fermi gas from the operator product expansion," Phys. Rev. A 84, 043603 (2011) arXiv:1106.6035 [cond-mat.quant-gas]]. 